\title{
Neurociência no tratamento dos transtornos depressivos
}

\author{
Dr. André Leandro K. Castanhede ${ }^{1}$ \\ Dr. Fabiano de Abreu Agrela Rodrigues ${ }^{2}$ \\ Paulo Fernando Lopes Dias Alves ${ }^{3}$ \\ Margieli dos Reis Alves ${ }^{4}$ \\ Dr. Francis Moreira da Silveira ${ }^{5}$
}

\section{RESUMO}

Como a neurociência pode tomar a frente no desenvolvimento de tratamento dos transtornos depressivos? Desse modo, faz se necessário entender que o transtorno neurobiológico da depressão, é um tema dos estudos no sentido de decifrar a mente humana, observando a complexidade do Sistema Nervoso Central , entendendo o que pode ser limitante durante as neurotransmissões, os prejuízos visualizados no SNC por meio das neuroimagens feitas internamente no cérebro, e claro, as possíveis medidas de intervenção tradicional e as novas descobertas que podem ser a chave para entender a neurociência da depressão. A modernização dos exames para investigação do sistema nervoso central (SNC) são grandes ferramentas que contribuem com a psiquiatria de modo geral. Os estudos científicos desenvolvidos atualmente consideram muito além dos aspectos mentais. Desta forma, a neurociência se insere no vasto campo dos transtornos cognitivas.

Palavras Chaves: neurobiologia da depressão; neurociências; sistema nervoso central; distúrbios cognitivos.

\footnotetext{
${ }^{1}$ Professor Orientador. Doutor Honoris Causa. Universidade San José, Doutor h.c. pela Faculdade Ciências Médicas FABIC, Formação em Naturopatia (Logos University Int., Gestão de Práticas Integrativas e Complementares, Mestre em Motricidade Humana - UCB-RJ. Professor Honoris Causa pela Logos University Int., Doctor in Health Science (D.L.) Logos University Int. e Universidade da Califórnia.

$2 \mathrm{PhD}$, neurocientista, mestre psicanalista, biólogo, historiador, antropólogo, com formações também em neuropsicologia, psicologia, neurolinguística, neuroplasticidade, inteligência artificial, neurociência aplicada à aprendizagem, filosofia, jornalismo e formação profissional em nutrição clínica - Diretor do Centro de Pesquisas e Análises Heráclito; Chefe do Departamento de Ciências e Tecnologia da Logos University International, UniLogos; Membro da Federação Européia de Neurociências e da Sociedade Brasileira e Portuguesa de Neurociências. Universidades em destaque: Logos University International, UniLogos, Nova de Lisboa, Faveni, edX Harvard, Universidad de Madrid.

${ }^{3}$ Neurocirurgião Intensivista e Médico Pesquisador Instituto Brasil Futuro.

${ }^{4}$ Enfermeira, pós-graduanda em Saúde Mental, Desenvolvimento Humano e Gestão das Emoções, PUC Paraná.

${ }^{5}$ Médico psiquiatra, especialista em dependência química. Membro da associação Brasileira de Neuropsiquiatria e Membro efetivo da associação Brasileira de Psiquiatria.
} 


\title{
Neuroscience in the treatment of depressive disorders
}

\begin{abstract}
How can neuroscience take the lead in developing treatment for depressive disorders? Thus, it is necessary to understand that the neurobiological disorder of depression is a subject of studies to decipher the human mind, observing the complexity of the Central Nervous System, understanding what can be limiting during neurotransmissions, the damage seen in the CNS through neuroimaging done internally in the brain, and of course, possible traditional intervention measures and new discoveries that may be the key to understanding the neuroscience of depression. The modernization of tests for investigation of the central nervous system (CNS) are great tools that contribute to psychiatry in general. Scientific studies currently carried out consider much beyond the mental aspects. In this way, neuroscience is inserted in the vast field of cognitive disorders.
\end{abstract}

Key Words: neurobiology of depression; neuroscience; central nervous system; cognitive disorders.

Artículo recibido: 05 octubre. 2021 Aceptado para publicación: 02 noviembre 2021

Correspondencia: Conflictos de Interés: Ninguna que declarar 


\section{INTRODUÇÃO}

Neurociência é a ciência que estuda o sistema nervoso, principalmente o cérebro. $\mathrm{O}$ entendimento de como o cérebro funciona tem se expandido para além de descrições anatômicas e fisiológicas. Em relação ao processo cerebral e seus detalhes iniciais, Martins (2017) considera que a estrutura do SNC é dividida em Lobo Frontal, tomada de decisões, prevenção e responsável pela fala; Lobo Temporal, responsável pelas funções auditivas, reconhecimento de faces e objetos; Lobo Parietal, responsável pela Linguagem, planejamento, sensações e orientação do corpo e o Lobo occipital, responsável pela visão. Com tantas mudanças existentes em cada momento estuda-se como o cérebro está relacionado com comportamento, pensamento e sensações.

Para entender profundamente o aprendizado e as dificuldades humanas, é necessário decifrar como o cérebro funciona. Como dito pela pesquisadora Hart (2002) Apud Ramos (2014) "Ensinar sem levar em conta o funcionamento do cérebro seria como tentar desenhar uma luva sem considerar a existência da mão". Parafraseando a autora, o corpo humano não funciona sem o cérebro e nem esse, sem o corpo. Portanto, o cérebro doente desenvolve um corpo doente e um corpo doente desenvolve um cérebro fraco em todos os aspectos.

Os princípios da neurotransmissão química do SNC são os Neurônios, Dendritos, Corpo celular, Axônio e fenda sináptica. Para um funcionamento normal do Sistema Nervoso Central esses princípios precisam estar saudáveis com boa comunicação. Os aspectos relacionados a Neurotransmissão se diferenciam entre uma síntese dos neurônios presente no terminal pré-sináptico, ser liberada em quantidade suficiente e possuir os sítios de captação e recaptação desse processo. (MARTINZ, 2017). Quando a comunicação com o SNC falha, alteram-se os seus princípios demonstrados pela ausência, diminuição ou excesso de neurotransmissão e/ou tempestades elétricas.

A Neurobiologia, considerada uma ciência que estuda o cérebro e a capacidade neuronal, abrange tais falhas na neurotransmissão, com o intuito de compreender anormalidades neurobiológicas que causam os transtornos psiquiátricos e neurológicos, entender o funcionamento normal e as alterações e compará-las. Estudos apontam que uma em cada cinco pessoas pode apresentar doença mental durante a vida e aproximadamente $4 \%$ da população sofre de transtorno mental crônico grave. É necessário saber quais áreas são 
relacionadas a esses transtornos para que seja possível pensar em intervenções no sentido de cuidar e tratar das alterações. (MARTINZ, 2017).

\section{JUSTIFICATIVA}

Essa pesquisa justifica-se ao buscar compreender o transtorno neurobiológico da depressão, analisando a importância dos estudos neurobiológicos no sentido de decifrar a mente humana, revelando a complexidade do SNC, as limitações que podem ocorrer na neurotransmissão, os prejuízos dessa, na comunicação interna no cérebro, as medidas de intervenção tradicionais e as novas descobertas que colaboram no sentido de entender a neurociência da depressão. Desse modo, evidencia-se a importância e a necessidade que a neurociência tem na expansão dos conhecimentos e estudos em relação aos transtornos neurais.

Ela contribui em nível geral e específico no aumento do acervo dos estudos científicos sobre a Neurobiologia na Depressão, relatando em sua trajetória temas como a depressão e os tratamentos neurobiológicos desenvolvido para os transtornos depressivos. Em nível institucional, enseja-se estudos neurobiológicos e construções cientificas e em nível pessoal, apresenta-se o conhecimento adquirido sobre a neurobiologia da depressão, comprovando cientificamente a eficácia e os avanços nos estudos, esperando que essa pesquisa seja usada como base cientifica no desenvolvimento de outros estudos científicos.

\section{REFERENCIAL TEÓRICO}

\subsection{Neurociência}

O termo Neurociência teve início em 1970, no entanto, as análises dos estudos sobre o cérebro humano fazem parte das questões filosóficas e gregas muito antes de Cristo, e tem se tornando ainda mais necessária atualmente. O principal interesse é investigar os objetivos do hipocampo na criação das memórias, a importância presente no sistema límbico no contexto emocional e o entendimento dos mistérios que se articulam na região frontal do cérebro em função a cognição, linguagem e escrita. (RAVAGNANI \& ALVES, 2019).

Nas análises sobre como cérebro humano aprende e suas ações no processo de movimento do corpo humano, cada ação é coordenada pelo SNC, através da neurotransmissão, desse modo, é possível que uma pessoa enfrente desafios, questões e dinâmicas, arte-cultura, e prazeres, e nesse caso, a neurotransmissão garante a completude do processo, o 
armazenamento do período e do que foi desenvolvido. Nesse sentido, nasce a importância das emoções e sentimentos no processo de aprender e entender as pautas no estudo do cérebro humano. (RAVAGNANI \& ALVES, 2019).

Por volta do ano de 1900, Harvey Cushing, o homem que atualmente é considerado "o pai da neurocirurgia “, desenvolveu com sucesso a primeira cirurgia de tumor cerebral. Em 1937, Walter Dandy realizou o primeiro recorte cerebral, ao qual, deram-lhe o nome de aneurisma. Comprometido e dedicado a compreender o sistema nervoso por meio de pesquisa e como isso pode afetar o atendimento clínico, Johns Hopkins traduziu e definiu Neurology e Neurosurgery em português - Neurologia e Neurocirurgia. (HOPKINS, 2019).

A nova história do Departamento de Neurocirurgia de Johns Hopkins conta o fascinante impacto em descobertas e tratamento inovadores. A equipe emergiu rapidamente como líderes em pesquisas e em invenções respectivamente sobre como o cérebro reage a lesões e o uso de neuronavegadores para orientar as cirurgias do cérebro. (HOPKINS, 2019).

Anos depois, os neurologistas e neurocirurgiões do instituto Johns Hopkins descobriram os traços, distúrbios e lesões do sistema nervoso, observando os problemas distintos que surgiam e, somente com cuidados intensivos e especializados, os pacientes poderiam ser salvos. São considerados pioneiros na primeira unidade dedicada de cuidados críticos neurológicos (NCCU) no país. (HOPKINS, 2019).

Segundo Lage (2010) a neuropsicologia pode ser definida como uma ciência aplicada que estuda a repercussão de disfunções cerebrais sobre o comportamento e a cognição e vem atualmente, ganhando importância nos estudos das desordens psiquiátricas. Por meio de alguns testes, percebe-se que ela é capaz de fornecer conhecimento sobre o potencial cognitivo global de um paciente, e propicia uma qualificação na natureza funcional, detalhando os déficits e os transtornos, analisando, comparando e qualificando os resultados que se obtém, permitindo a possibilidade de correlações anatômica-funcional refinadas. Desse modo, a neuropsicologia completa o diagnóstico clínico com informações concluídas de exames complementares, mensura a atividade eletrogênea, a atividade metabólica e a neuroimagem, criando uma conexão entre diagnóstico e o quadro clínico do paciente.

Para Rozenthal et al (2004) “A neurobiologia da depressão conheceu, nesta última década, avanços sem precedentes, que vão desde alterações macroscópicas até ao nível 
molecular". Um estudo da base neuronal representada pelas redes neuronais com a forma intracelular, visando a observância do comportamento e da experiência. A neurobiologia da depressão conheceu, nesta última década, avanços que mudaram toda a forma de diagnóstico desde as alterações macroscópicas até o nível molecular, mudança na percepção genômica e a interação com o ambiente, e claro, os fenômenos de plasticidade neuronal e das modulações endócrinas e imunológicas.

Segundo a autora "o estudo de alterações neuro funcionais e neuropsicológicas ainda está longe de elucidar os fatores causais referentes aos processos mentais". Entende-se que a estrutura do SNC é frágil e por isso, pode ter interferências em aspectos genéticos e constitucionais, os transtornos mentais. Para ela, "a demonstração de que o aprendizado é acompanhado por modificações da eficácia das conexões neurais tem provocado a revisão dos conceitos sobre as relações entre os processos sociais e biológicos na determinação dos padrões de comportamento" (ROZENTHAL et al., 2004).

As rotinas diárias podem provocar um enfraquecimento efetivo das conexões sinápticas. Em algumas situações são essas rotinas que fortalecem essas conexões, sendo esse um objeto de estudo relevante na busca de técnicas terapêuticas não-medicamentosas com o objetivo de estímulo das disfunções clínicas observadas em testes. Os transtornos nos idosos e nos adultos, por exemplo, causam sérios comprometimentos, funcionais e diversas recaídas recorrentes, o paciente demora para alcançar certa estabilidade ou até mesmo para reagir aos tratamentos. Nesse sentido, Rozenthal et al (2004) afirma que "a neuropsicologia deve ser considerada como um importante instrumento na compreensão dos transtornos mentais, sendo de grande utilidade no processo de estruturação das intervenções terapêuticas mais diretivas para os déficits observados".

\subsection{Transtorno da depressão}

Atualmente, a depressão tem sido considerada o grande mal que afeta a sociedade civil. De modo recorrente, toda pessoa pode afirmar já ter visto ou percebido alguém com dificuldades em relação à oscilação de humor. Estimativas da Organização Mundial da Saúde apontam que, no mundo todo, pelo menos 350 milhões de pessoas convivam com a depressão, sendo que um em cada três pacientes não respondam aos remédios tradicionais. Essa enfermidade está classificada entre as doenças degenerativas que desenvolve mortes prematuras. Apesar do tempo que se conhece a depressão, ainda não 
existem estudos que expliquem esses processos psíquicos e emocionais (RODRIGUES, 2000; DELOUYA, 2001; TEODORO, 2009).

Ao definir depressão, nos deparamos com o conceito que abrange um transtorno mental, apresentado por sentimentos que se misturam e, ao mesmo tempo, faz com que a pessoa sinta aflição orgânica, psicológica, ambiental e espiritual (RODRIGUES, 2000; DELOUYA, 2001; TEODORO, 2009), considerada como uma angústia que impacta diretamente no humor seguida de perda de interesse, de prazer e alegria na vida. Diante da angústia e aflição, a pessoa desenvolve dores físicas, psicológicas, na alma, num ciclo de sofrimento tão intenso que parece não ter fim.

A depressão é um turbilhão de situações que perseguem uma pessoa de uma só vez, é como se todas as frustações familiares, profissionais, conjugais, acadêmicas, todas as palavras ouvidas, todas as críticas, reprovações e tudo de ruim que aquela pessoa já viveu, viesse à tona com uma força letal que a atinge todos os aspectos e, nesse momento a pessoa é tomada por desânimo, isolamento e desinteresse por tudo o que um dia foi importante para ela. (RODRIGUES, 2000; DELOUYA, 2001; TEODORO, 2009).

É preciso esclarecer que existe no senso comum, ideias preconcebidas sobre a depressão. O paciente depressivo muitas vezes é visto como um preguiçoso, acomodado que não tem o que fazer e "está cheio de frescuras e não me toque", cujas afirmações são das pessoas próximas àquele indivíduo em vez de apoiá-lo e prestar socorro. Decorrente disso, o depressivo precisa vencer esse tipo de agressão que pode piorar seu quadro.

Em alguns casos, os pacientes demonstram que não precisam se tratar, quando apresentam certa melhora de humor, com euforia, animação e ansiedade. Porém, sem o tratamento correto e a devida atenção médica esse paciente tenderá a ter novas recaídas que fará com que a depressão apareça novamente. Esse comportamento é chamado de "mania" pelos estudiosos e enganam muitas pessoas que em seguida, aparecem piores do que o primeiro estado, porque achavam que não necessitavam de tratamento. (RODRIGUES, 2000; DELOUYA, 2001; TEODORO, 2009).

Os sintomas encontrados na depressão podem incidir sobre as áreas do humor, da cognição e pensamento, em aspectos somáticos, na expressão corporal e vida social. Dentro do humor intensificam-se a tristeza, emotividade, angústia, irritabilidade, ansiedade, anedonia e desmotivação. Nos sintomas cognitivos percebe-se uma queda intelectual, falta de fé, sentimento de abandono e rejeição, desejo incessante de morte 
inferioridade, falta de sentido, baixa autoestima, autoimagem negativa, pessimismo, desvalorização da vida, sentimento de culpa e ideias suicidas. Nos sintomas somáticos percebemos a hipersonia, a insônia, a perda de apetite, diminuição da libido, falta de interesse sexual e baixa no sistema imunológico. Na expressão corporal observa-se mudanças posturais como: cabeça baixa, peito embutido, coluna curvada, não consegue fixar "olho no olho", apresenta olhar desvitalizado, despreocupação com a aparência pessoal, respiração superficial e movimentos lentos e contidos. Sua vida social é isolada, sem interesse nos estudos, em passeios e no trabalho. (RODRIGUES, 2000; DELOUYA, 2001; TEODORO, 2009).

De acordo com DSM 5, 2014, o Manual Diagnostico e Estatístico de Transtornos Mentais da Associação Americana de Psiquiatria, em sua quinta edição, aponta critérios diagnósticos que possibilitam a clara distinção entre depressão maior de reações normais a determinados eventos como, por exemplo a morte de um ente querido (chamada de depressão reativa e não constitui um transtorno psiquiátrico; ao menos que os sintomas sejam desproporcionais ao evento ou significantemente prolongados). A depressão maior caracteriza-se por um ou mais episódios depressivos, com pelo menos duas semanas de humor deprimido ou perda por interesse na maior parte das atividades (anedonia = perda da sensação de recompensa ou incapacidade em experimentar prazer em qualquer coisa), acompanhados de ao menos quatro sintomas adicionais, que podem incluir sentimentos de esperança, desvalia, culpa; associados a alterações de apetite e sono, fadiga, retardo ou agitação psicomotora, dificuldade de concentração e raciocínio e pensamentos recorrentes sobre morte, com ou sem tentativas de suicídio.

O ideal é que a família, ao perceber os sintomas citados acima, não espere uma piora, mais que procure logo ajuda profissional e orientação. Ignorar a depressão pode acarretar a perda do ente querido e em alguns casos, a demora em buscar ajuda médica, modifica por completo a vida desses pacientes. Pensando em um tratamento efetivo para a depressão, esse trabalho deseja observar como o tratamento homeopático pode colaborar no tratamento da depressão. (RODRIGUES, 2000; DELOUYA, 2001; TEODORO, 2009).

\subsection{Tratamentos dos Transtornos Depressivos}

As neuroimagens revelam os circuitos cerebrais, principalmente os que regulam o humor, estão desregulados na pessoa que tem depressão. Geralmente é percebido que no interior 
do cérebro, a amígdala é altamente responsiva pelos estímulos salientes tais como recompensas e ameaças potenciais.

Barenboin (2020) afirma que os estudos atuais refutam a explicação que a depressão está relacionada somente com a falta de serotonina no cérebro. Segundo o autor a refutação se dá baseada "no fato de que a maioria dos antidepressivos aumentam a disponibilidade de serotonina nas sinapses cerebrais. Se este fosse o caso, a depressão seria comparável a uma disfunção endócrina: tal órgão não está produzindo quantidade certa de tal hormônio, então apenas devemos repor esta substância que está em baixos níveis”. No caso do hipotireoidismo, funciona assim. No entanto, as alterações neurobiológicas ligadas a depressão são bem mais complexas do que a simples reposição de serotonina da mesma forma como se dá mecanismo de ação dos antidepressivos

Em concordância, Lage (2010) fala que "o humor flutua naturalmente e todo mundo experimenta altos e baixos na vida, os casos de mudanças de humor de longa duração podem se tornar debilitantes e podem prejudicar a vida". Essa pessoa pode comprometer seu trabalho, os relacionamentos familiares e com amigos. Uma média de 10 por cento das pessoas podem vir a sofrer de depressão, pois ela é um transtorno de humor caracterizado por sintomas conhecidos como tristeza, angústia, desânimo, cansaço excessivo e perda do interesse em atividades prazerosas, fadiga e ansiedade.

Para Barenboim (2020), “na depressão, a amígdala é hiperativa e responde excessivamente a eventos negativos, no entanto, a amígdala se conecta a um conjunto de regiões cerebrais que dispara a resposta fisiológica e comportamental aos estímulos emocionais". Em sua estrutura está o córtex pré-frontal medial, o núcleo accumbens, o hipocampo e a ínsula. Deve-se considerar que o hipocampo envolve a formação da memória e o córtex pré-frontal, os efeitos do estresse. $\mathrm{O}$ autor ainda explica que "o córtex pré-frontal medial está envolvido na regulação de quão fortemente reagimos a estímulos. Tratamentos com medicamentos antidepressivos, terapia cognitivo-comportamental e eletroconvulsoterapia afetam a estrutura e a função dessas e de outras regiões do cérebro" (BARENBOIN, 2020).

As alterações físicas no cérebro, incluindo a atrofia do hipocampo são o motivo da suscetibilidade das pessoas depressivas sofrerem de fortes níveis de estresse. São mudanças que podem causar respostas inadequadas para eventos emocionais. Devido as novas rotinas empregadas pelas pessoas uma boa parte do aumento de casos depressivos 
está relacionada aos níveis de estresse, não se pode dizer que toda depressão humana seja desencadeada por estresse, porém serve como gatilho para explicar a biologia da depressão. De acordo Barenboim (2020),

“Os modelos animais com camundongos são fundamentais para nos ajudar a entender as mudanças celulares e moleculares subjacentes a depressão e a desenvolver melhores tratamentos. Apesar de ser impossível saber se um camundongo está deprimido, os roedores submetidos a estresse crônico apresentam alguns sintomas semelhantes aos seres humanos deprimidos tais como ansiedade, menos interação social e menos interesse em atividades prazerosas" [...] "Tal como acontece com os seres humanos, o estresse crônico em camundongos pode levar a atrofia do hipocampo e do córtex pré-frontal. Estudos também demonstraram plasticidade neuronal alterada em várias regiões do cérebro, incluindo o córtex pré-frontal, hipocampo, a amígdala, e núcleo accumbens".

De acordo Lage (2010), “em um hipocampo saudável, experiências podem levar a mudanças nas conexões entre os neurônios, resultando em aprendizado. Estas alterações são denominadas neuroplasticidade. Desse modo a neurotransmissão pode ocorrer da forma correta. No entanto, o estresse crônico reduz a plasticidade, os cérebros saudáveis continuam a produzir novos neurônios em uma parte do hipocampo e esses novos neurônios lentamente modulam e integram os seus circuitos.

Nesse processo, acontece um forte efeito entre a atividade ou o comportamento do hipocampo. Os neurônios são afetados significativamente pelo estresse podendo até mesmo reduzir, nos cérebros estressados. Segundo Lage (2010) as neurotrofinas compõem a redução da plasticidade o que pode ser prejudicial na capacidade do hipocampo de regular adequadamente o nível de estresse, desse modo, iniciaria um ciclo vicioso no qual estresse acarreta em mais estresse.

Lage (2010) afirma que a plasticidade pode ser reduzida em outras partes do cérebro, e o conjunto destas alterações pode contribuir para outros sintomas da depressão, como por exemplo, a anedonia. Barenboim (2020) considera que a maioria dos antidepressivos 
podem aumentar rapidamente a quantidade de neurotransmissores como serotonina e norepinefrina nas sinapses.

Lage (2010) afirma que "no entanto, a melhora dos sintomas em pacientes e em camundongos leva semanas para ocorrer". A resposta tardia dos medicamentos não é totalmente compreendida, e o tratamento com antidepressivos geralmente costuma ser longo, mas, pode estimular mudanças de humor, reduzir o estresse crônico aumentar a expressão de neurotrofinas e reiniciar a plasticidade do hipocampo.

Em tratamentos considerados não-químicos recomendados para a depressão, usa-se a eletroconvulsoterapia. Ela pode promover plasticidade do hipocampo por exemplo, em camundongos. $\mathrm{O}$ antidepressivo reduz alterações causadas pelo estresse em outras áreas do cérebro, incluindo o córtex pré-frontal, circuitos de recompensa e diferentes tratamentos podem atingir diferentes regiões para melhorar os sintomas (BARENBOIN, 2020).

Estudos inconclusivos sobre uma droga chamada cetamina comprovam seu rápido efeito antidepressivo: bloqueia a transmissão sináptica, no intuito de ativar o número de vias de sinalização e aumentar a expressão de neurotrofinas. Desse modo, as alterações moleculares podem resultar em um aumento da plasticidade no córtex pré-frontal e no hipocampo que contribui para os efeitos terapêuticos da cetamina. Ao estudar as mudanças no cérebro causadas pela depressão e como os antidepressivos como a cetamina reagem, os pesquisadores podem encontrar novas drogas que atuem no tratamento de forma mais rápida, sendo mais específicas que os tratamentos disponíveis (BARENBOIN, 2020; LAGE, 2010).

Diretrizes clínicas baseadas em evidências pela Rede Canadense de Tratamentos de Humor e Ansiedade (CANMAT) realizou uma revisão das diretrizes de 2009, atualizando as evidências e recomendações. O escopo das diretrizes de 2016 continua sendo o gerenciamento do transtorno depressivo maior (TDM) em adultos, com um público-alvo de psiquiatras e outros profissionais de saúde mental. (CANMAT, 2016).

Desse modo, tratamentos farmacológicos baseados em evidências estão disponíveis para o tratamento de primeira linha de TDM e para o manejo da resposta inadequada. No entanto, dadas as limitações da base de evidências, o manejo farmacológico do TDM ainda depende da adaptação dos tratamentos ao paciente. 
No que tange a genética: há influência dos endofenótipos nos sintomas psiquiátricos ou dimensões da psicopatologia que transpõem as numerosas síndromes psiquiátricas. Isso não necessariamente respeita os critérios do Manual Diagnóstico e Estatístico de Transtornos Mentais da Associação Americana de Psiquiatria (DSM) ou da Classificação Internacional de Doenças (CID). O futuro da Psiquiatria, está no entendimento da combinação de endofenótipos com circuitos cerebrais hipoteticamente defeituosos, regulados por genes, pelo ambiente e por neurotransmissores (Stahl, 2014).

É fundamental identificar a necessidade dos pacientes com transtorno depressivo, pesquisando e buscando tratamentos que melhorem a qualidade de vida por meio de estratégias compensatórias e preservando a individualidade de cada caso clínico. Tais estudos buscam integrar algumas terapias para a resolução dos problemas sendo eficaz na redução dos sintomas depressivos e na melhoria do desempenho das atividades da vida diária, como alternativa terapêutica importante para a população que permanece sintomática e sofrendo muito. Recentemente, pesquisa com estimuladores implantados, por neurocirurgiões, no cérebro de humanos, parecem promissores.

\subsection{Terapia EDMR}

Segundo Dr. Mesquita (2020) uma terapia que está sendo comprovada cientificamente como funcional no tratamento de muitos transtornos neurais e perturbações é a Dessensibilização e Reprocessamento Cerebral através do Movimento Ocular (EMDR, Eye Movement Desensitization and Reprocessing). O autor considera como "uma terapia breve, de base fisiológica, que promove a comunicação entre os dois hemisférios cerebrais". Em meados dos anos 80 a EMDR foi um dos métodos psicoterapêuticos mais investigados nos EUA, e tem sido recomendação especial da Associação Americana de Psiquiatria. Os estudos comprovam a sua eficácia em várias intervenções: fobias, transtornos do pânico, depressão e perturbações psicossomáticas.

De acordo Almeida (2017) “o Reprocessamento e Dessensibilização através de Movimentos Oculares (EMDR) surgiu há 30 anos como modelo de intervenção na prática psicoterapêutica”. Nessas 3 décadas, aumentou-se as investigações em torno dessa terapia considerando-a como uma abordagem literária e prática com possibilidades de crescimento. A medicina vibracional, a substância psicodélica associadas à psicoterapia especifica entre outras técnicas, comprovadamente são eficazes. 
No sentido mais amplo, o EMDR procura aliviar o sofrimento humano e assistir aos indivíduos e à sociedade na realização integral de seu potencial para o desenvolvimento, minimizando os riscos de dano durante sua aplicação. Para o paciente, o propósito do tratamento com o EMDR é obter os mais profundos e eficazes efeitos no mais curto período de tempo, mantendo estabilidade dentro dos sistemas familiar e social. Quando a informação armazenada nas redes de memória relativas a uma experiência traumática ou perturbadora não é completamente processada, torna-se possível o surgimento de reações disfuncionais.

O processamento para estados adaptativos resulta em aprendizagem, alivio da perturbação emocional e somática, e em possibilidade de obter respostas adaptativas e integradas.

Um outro conhecimento abordado por Almeida (2017) trata a respeito do interesse da comunidade científica nos últimos 15 anos nas consideradas "crescente nas potencialidades do Mindfulness: um "antigo produto, com um novo rótulo". O autor considera que a "EMDR e Mindfulness possuem vasto potencial de integração" promovendo possíveis mudanças terapêuticas e processos psicológicos. Desse modo, o EMDR juntamente com o Mindfulness e outras terapias supracitadas, podem ser positivos no estímulo de respostas durante o tratamento. Essa relação entre EMDR e Mindfulness pode ser considerada como tratamento não linear desenvolvido para condutas terapêuticas e não-terapêuticas. (ALMEIDA, 2017).

\section{METODOLOGIA}

Segundo Pizanni (2012), entende-se por pesquisa bibliográfica a revisão da literatura sobre as principais teorias que norteiam o tema científico a que se deseja tratar. O estudo teve como metodologia uma revisão bibliográfica sistemática com pesquisa em livros físicos e online, revistas científicas digitais buscando-se levantar material para a pesquisa. A pesquisa bibliográfica propiciou a identificação, classificação e organização dos documentos utilizados. A pesquisa bibliográfica foi desenvolvida a partir de materiais publicadas em livros, artigos, dissertações e teses.

Quanto à abordagem, a pesquisa se caracteriza por qualitativa que significa que a análise depende do pesquisador como ferramenta de interpretação assim, "É baseada na interpretação e no significado que carregam, ou no significado atribuído pelo pesquisador, 
dada a realidade em que os fenômenos estão inseridos. Considera a realidade e a particularidade de cada sujeito objeto da pesquisa". (NASCIMENTO, 2016).

Neste sentido, os resultados e a análise dos dados foram realizados a partir do arcabouço teórico, onde foram considerados os aspectos apresentados por cada autor e daí realizado um paralelo a fim de responder ao objetivo geral deste estudo que, como já citado, foi o de identificar em publicações científicas a contribuição da homeopatia no tratamento de pessoas que sofrem com depressão, para a melhoria da sua qualidade de vida do portador.

\section{RESULTADOS}

De um total de aproximadamente 15 documentos levantados, foram excluídos aqueles sem referência confiável de origem e autoria, com temas repetidos e textos comuns a outros, restando selecionados 10 que se encontram devidamente referidos no texto e compõem a bibliografia deste artigo. Com base nesse material, foi levantado tema da Neurobiologia da Depressão. A relevância do tema corrobora com os estudos de neurociência e chega-se ao entendimento que o método de tratamento dos transtornos depressivos por meio da neurociência vem colaborado para os resultados positivos nos quadros de depressão, combatendo os sintomas, e a depender do quadro clinico, à cura dos pacientes acometidos por essa enfermidade.

\section{CONSIDERAÇÕES FINAIS}

Os aspectos neurobiológicos dos transtornos mentais e, no caso específico desta revisão, da depressão, são importantes para a aproximação dos campos da neurociência ao da psiquiatria. Não se pode perder a dimensão crítica dos achados encontrados, havendo várias questões e controvérsias que ainda continuam em aberto, ainda é necessário avançar em tratamentos melhores, mais rápidos e eficientes.

Notório que a depressão é um quadro complexo tanto do ponto de vista sintomatológico quanto etiológico, determinante de interação entre predisposição genética, problemas no desenvolvimento como perda precoce dos pais, abuso ou negligência na infância e a presença de eventos estressantes na vida.

Salienta se que exposição ao estresse crônico eleva os níveis de glicocorticoides, o cortisol e resulta em alterações plásticas no Sistema nervoso central, como a redução da expressão de fatores neurotroficos (como o BDNF - fator neurotrofico derivado do cérebro) e a redução da arborização dendrítica. Consequentemente, o estresse reduz a neurogenese no hipocampo adulto. 
Os estudos de terapias como a EDMR estão ganhando atenção durante esse processo na avaliação dos transtornos, principalmente por proporcionar alívio. Desse modo, também se torna válido a análise e o aprofundado estudo das medidas integrativas como por exemplo terapias EDMR e possíveis tratamentos homeopáticos dentre outros.

No entanto, vários aspectos estão sendo compreendidos pelos clínicos e cientistas, permitindo uma melhor compreensão do estado crítico nos transtornos de humor e abrindo caminhos para a busca de técnicas mais eficazes de diagnóstico, tratamento, prevenção de eventuais sequelas cognitivas e suicídio.

\section{REFERÊNCIAS}

ALMEIDA, Francisco. EMDR E Mindfulness como Abordagem Integrativa em Psicoterapia: um estudo de caso. Repositório FPCUP, out,2017. Disponível em <https://repositorio-aberto.up.pt/bitstream/10216/108501/2/227283.pdf> Acesso em: 29 de jul.2021.

AMERICAN PSYCHIATRIC ASSOCIATION - APA. Manual diagnóstico e estatístico de transtornos mentais: DSM-5. Porto Alegre: Artmed, 2014.

BAREMBOIM, Ivan. A neurobiologia da Depressão e do seu tratamento. OHR Psiquiatria. 2020. Disponível em <https://clinicaohrpsiquiatria.com/aneurobiologia-da-depressao/> Acesso em: 29 de jul. 2021.

DELOUYA, Daniel. Depressão: Clínica Psicanalítica. São Paulo: Casa do Psicólogo $5^{\text {a }}$ ed. 2001. Disponível em <https://books.google.com.br/books?hl=pt$\mathrm{BR} \& 1 \mathrm{r}=\& \mathrm{id}=2 \mathrm{qpahi} 1$ esjoC $\&$ oi=fnd\&pg=PA1\&dq=origem + da + depress $\% \mathrm{C} 3 \% \mathrm{~A}$ 3o\&ots=i9GhrnVv8p\&sig=zSNRnpWCIXAnZliKs84Vvg23Ao8\#v=onepage\&q =origem $\% 20 \mathrm{da} \% 20 \mathrm{depress} \% \mathrm{C} 3 \% \mathrm{~A} 3 \mathrm{o} \& \mathrm{f}=$ false $>$. Acesso em 18 de jul. 2021.

LAGE, Jorge. Neurobiologia da Depressão. Repositório FMUP: Porto, 2010. Disponível em $<$ https://repositorioaberto.up.pt/bitstream/10216/53466/2/Neurobiologia\%20da\%20Depresso.pdf>. Acesso em: 29 de jul. 2021.

MARTINS, Daiane. Curiosidades da Neurobiologia dos transtornos psiquiátricos. Vida mental, Dez, 2017. Disponível em <https://www.youtube.com/watch?v=pugMwQpiFLY>. Acesso 28 de jul. 2021. 
MESQUITA, Fernando. Terapia EDMR. 2020 Disponível em <https://www.fernandomesquita.net/sobre-mim> Acesso 29 de Jul. 2021.

NEUROCIRURGIÃO SP. Surgimento de Neurologia. 2019 Disponível em $<$ https://neurocirurgiaosp.com/neurologia-e-neurocirurgia-origem>. Acesso 28 de jul. 2021.

RAMOS, Ângela. Neurobiologia da Aprendizagem. Taguatinga, 2014. Disponível em <https://pt.scribd.com/document/351696490/Neurobiologia-Da-Aprendizagem>. Acesso 28 de jul. 2021

RAVAGNANI, P; ALVES, J. Os Recursos Da Neurociência No Âmbito Da Educação. 2019 Disponível em <https://pt.scribd.com/document/400943748/Freire-eneurociencias-pdf>. Acesso 28 de jul. 2021.

RODRIGUES, Maria. O Diagnóstico de depressão. Revista Scielo do Brasil. São Paulo, USP, 2000. Disponível em <https://www.scielo.br/j/pusp/a/pLGJppkNXcmwHdpQ5T3T5xQ/abstract/?lang $=\mathrm{pt}>$. Acesso em 19 de jul. 2021.

Stahl SM. Psicofarmacologia: bases neurocientíficas e aplicações práticas. 4. ed. Rio de Janeiro: Guanabara Koogan; 2014.

TEODORO, Wagner. Depressão, corpo, Mente e Alma. Uberlândia- MG: 2009. Disponível em <https://books.google.com.br/books?hl=pt$\mathrm{BR} \& \mathrm{lr}=\& \mathrm{id}=\mathrm{jzx} 0 \mathrm{LDwRW}-$ oC\&oi=fnd\&pg=PA87\&dq=surgimento + da + depress $\% \mathrm{C} 3 \%$ A3o\&ots $=009$ NFLe eib\&sig=soPS4cAhoHpywyz4cnJepF9gBwQ\#v=onepage \&q\&f=false>. Acesso dia 17 de jul. 2021.

ROZENTHAL; LAKS; ENGELHARDT. Aspectos Neuropsicológicos da Depressão. Revista Scielo ago., 2004. Disponível em: https://www.scielo.br/j/rprs/a/CLYL7Tmqw7vjWbCRJndzMSJ/?lang=pt\&forma $\mathrm{t}=$ pdf $>$. <Acesso em: 29 de jul. 2021>. 